\title{
Statistical Analysis of VLSI Using TCAD
}

\author{
Naoyuki Shigyo \\ System LSI Design Division, Toshiba Corporation Semiconductor Company \\ STE Bld., 2-5-1, Kasama, Sakae-ku, Yokohama 247-8585, Japan \\ E-mail: naoyuki.shigyo@toshiba.co.jp
}

\begin{abstract}
Statistical process fluctuations influence upon device and circuit performance of VLSI with device miniaturization. Thus, robust process, device and circuit designs are needed. This article describes the statistical analysis of MOSFET and interconnect using Technology CAD (TCAD).
\end{abstract}

\section{Introduction}

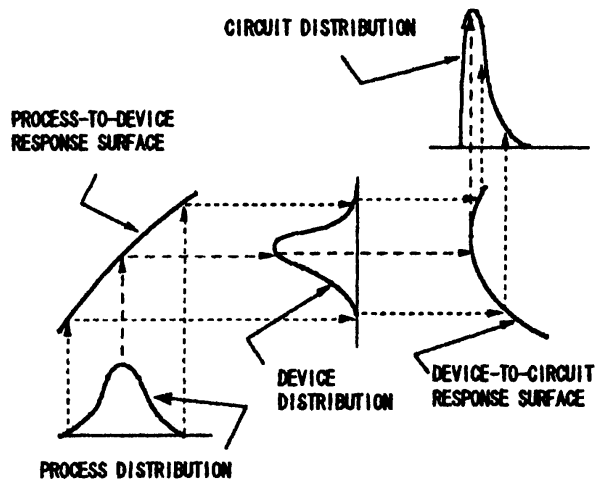

Fig. 1 Process fluctuation and circuit performance [2]

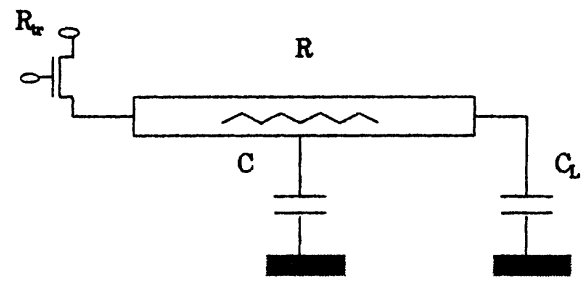

Fig. 2 Variability of device and interconnect.

The dependence of the parametric yield of VLSI on statistical process fluctuations such as a critical dimension (CD) and an ion implantation, has been increased. The statistical simulation is needed for robust designs of process, device and circuit [1].

Fig. 1 shows a schematic explanation of the relation between the process fluctuation and circuit performance [2]. The process fluctuation is related to device performance through a process-to-device response surface. The device performance is related to circuit performance throügh a device-to-circuit response surface. Process/device simulators can be used for representing the process-to-device response surface. Also, a circuit simulator is used for the device-to-circuit response surface.

This article presents TCAD based statistical analysis for the variability of device and interconnect, as shown in Fig. 2. The circuit delay $t_{\text {delay }}$ is approximated as $2.3 R_{t r} C+$ $R C$ where $R_{t r}$ is the MOSFET resistance [3]. In the case of short interconnect length 
$L, \mathrm{R}_{\mathrm{t}} \mathrm{C}$ dominates $\mathrm{t}_{\text {delay }}$. On the other hand, the interconnect $\mathrm{RC}$ delay dominates $\mathrm{t}_{\text {delay }}$ for long $L$. In Section 2, the worst case circuit model for MOSFET and problems for the statistical simulation are described. The interconnect variability is treated in Section 3.

\section{Device Variability: MOSFET}

\subsection{Worst case circuit model}

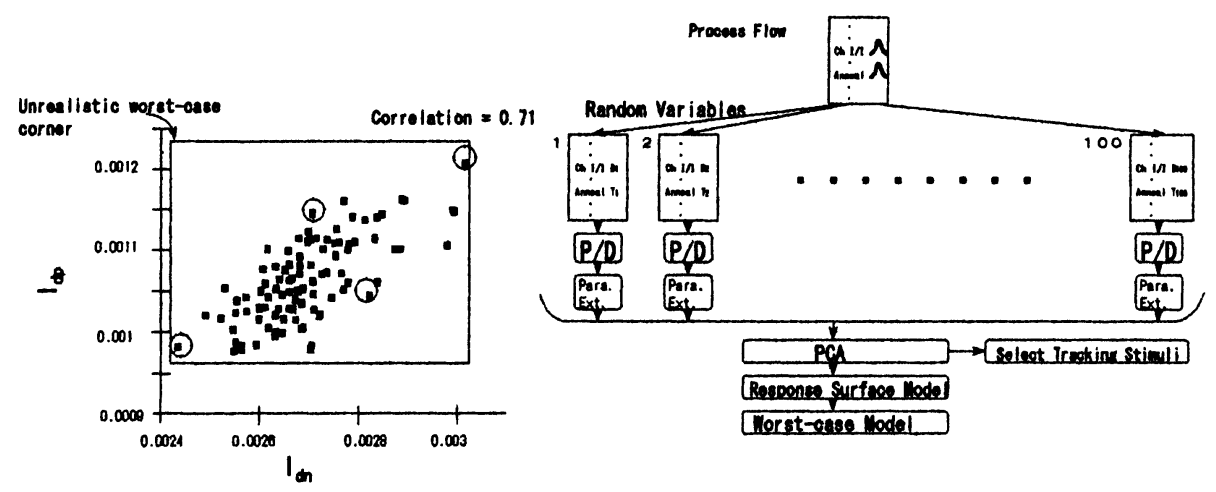

Fig. 3 Unrealistic worst-case corners [4].

Fig. 4 Schematic explanation for statistical analysis and generation of worst-case models [5].

Fig. 3 shows a scatter plot of the $n$-ch and $p$-ch saturation drain current, $I_{d n}$ and $I_{d p}$ [4]. A circuit designer has used min-max worst-case corner models. In this example, however, there was a strong correlation between $\mathrm{I}_{\mathrm{dn}}$ and $\mathrm{I}_{\mathrm{dp}}$, whose correlation coefficient was 0.71 . Thus, the upper-left and lower-right corners were unrealistic worst-case corners.

Fig. 4 shows a schematic explanation for the statistical analysis and generation of worst-case BSIM3v3 models [5]. Before Monte Carlo (MC) runs, process conditions for a simulation were assigned separately to each process step using random numbers with assuming process fluctuations of the normal distribution. The number of $\mathrm{MC}$ runs is related to the accuracy [6].

MC runs resulted in 97 sets of BSIM3v3 parameters. At each run, a set of $144(=72$ $\times 2$ ) model parameters for $n$-ch and p-ch MOSFET's was extracted. Hence, 97 points which corresponded to the extracted parameter sets, were placed in a 144dimensional space; a BSIM3v3 space. Independent factors were selected using the principal component analysis (PCA) [7]. Then, tracking stimuli were selected from the process variables, which had strong correlation to the factors. Finally, the response surface model (RSM), hence the worst-case models were generated as combinations of the tracking stimuli using the values of $\sigma$ for the process variables. It is noted that the conventional method gives the worst-case models in the sense of the 
corners of the process fluctuations, so that they do not always correspond to those for the circuit performance, as shown in Fig. 1.

Table 1 shows 11 factors, which were needed to cover the $80 \%$ of the BSIM3v3 space. An increase in the number of factors $m$ enlarges the coverage of the BSIM3v3 space. However, this results in an increase in the worst-case corners, hence, the number of required circuit simulations. For example, $128\left(=2^{11}\right.$ -4) circuit simulations are required even using the design-of-experiments (DOE) of resolution V. For a large circuit, a reduction of $m$ is important to decrease the circuit simulation time.

The total coverage of BSIM3v3 space using factors 1,2 and 3 was only $43 \%$. In order to supplement the small coverage, the factors were selected not by its coverage, but by its sensitivity to the performance of a small but critical circuit. The sensitivity analysis was carried out for reducing $\boldsymbol{m}$. A ring-oscillator was used as an example.

Fig. 5 shows the sensitivities of the factors to the delay time tpd with \pm $3 \sigma$ process fluctuations. The characters of the lateral axis indicate the sign of each factor. For example, folt means that factor 1 was changed $+3 \sigma$ from the nominal value. The short-channel effect was pronounced in the case of folt. Factors 2, 1, and 8 had higher sensitivities to tpd, where the tracking stimulus for factor $\mathbf{8}$ was the gate oxidation temperature. On the other hand, factor 3 (LDD spacer) had a small contribution to tpd. This is because LDD spacer is related to
Table 1 Tracking stimuli and coverage of BSIM3v3 space for the factors [5].

\begin{tabular}{|c|c|c|}
\hline Name & Triatding Stimulus & $\begin{array}{c}\text { Covernge of BSIMB } \\
\text { space }[\%]\end{array}$ \\
\hline $\begin{array}{l}\text { Pactor } 1 \\
\text { Pactor } 2 \\
\text { Pactor } 3 \\
\text { Pactor } 4 \\
\text { Pactor } 5 \\
\text { Pactor } 6 \\
\text { Pactor } 7 \\
\text { Factor } 8 \\
\text { Factor } 9 \\
\text { Pactor } 10 \\
\text { Pactor } 11 \\
\end{array}$ & 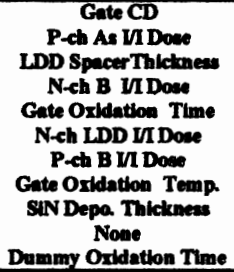 & \begin{tabular}{|l|}
19 \\
12 \\
12 \\
8 \\
7 \\
6 \\
4 \\
4 \\
4 \\
3 \\
2 \\
\end{tabular} \\
\hline
\end{tabular}

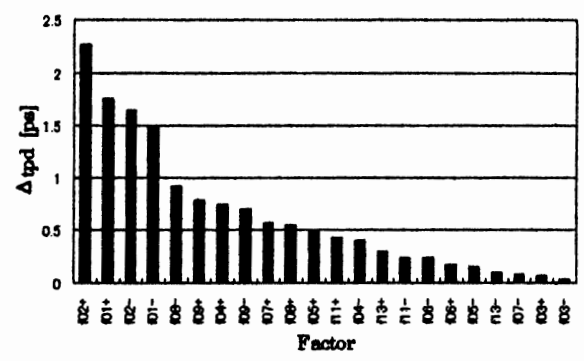

Fig. 5 Sensitivities of factors to delay time tpd [5]. $\mid \mathrm{VDS}_{\mathrm{DS}}=3.3 \mathrm{~V}, \mathrm{~W} / \mathrm{L}=5 / 0.3$ for $\mathrm{n}-\mathrm{ch}$, and $W / L=10 / 0.3$ for $p-c h$.

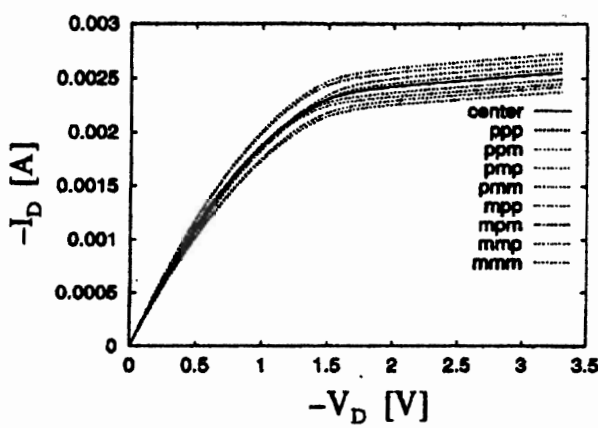

Fig. $6 \mathrm{ID}_{\mathrm{D}}-\mathrm{V}_{\mathrm{D}}$ characteristics of $\mathrm{p}$-ch MOSFET's using nine BSIM $3 \mathrm{v} 3$ worst-case models [5]. $V_{G S}=-3.3 \mathrm{~V}, V_{B S}=0 \mathrm{~V}$ and $W / L=10 / 0.3$. 
both the source/drain sheet resistance and the overlap capacitance. There was a tradeoff between the two, so that factor 3 had small influence on tpd. Factors 1, 2, and 8 were selected for reducing $m$. In general, the factor with high coverage of the BSIM3v3 space does not always correspond to the high sensitivity to the circuit performance.

Fig. 6 shows the p-ch $I_{D}-V_{D}$ characteristics of the nominal and $2^{3}$ worst-case models using factors 1,2 , and 8 , based on $\pm 3 \sigma$ process fluctuations. Factor 1 (CD) had large influence on both $I_{d n}$ and $I_{d p}$. Factor 2 (p-ch As $I / I$ dose) had largest influence on $I_{d p}$ due to the buried-channel structure of p-ch MOSFET's $(L=0.3 \mu \mathrm{m})$.

\subsection{Tasks for statistical simulation}

The following list describes problems for realizing the statistical simulation.

(1) Definition of the worst-case model in the sense of circuit performance [8].

(2) Estimation of the standard deviation $\sigma$ of the process fluctuation.

(3) TCAD predictability.

(4) Numerical noise such as the meshing noise [9]

The problem (4) is treated in this article. The meshing noise is very crucial problem for the sensitivity and statistical analysis.

Fig. 7 shows the threshold voltage $V_{\text {th }}$ as a function of the channel length $L$ [9]. There were discontinuities in $V_{\text {th. }}$. This is so-called meshing noise. The meshing noise is due to the change in the potential barrier between the source and channel, which is induced by the abrupt change in mesh points along the lateral direction. The default mesh was automatically generated. Hence, the abrupt change in the number of mesh points at some $L$ caused these $V_{\text {th }}$ discontinuities. There were only five mesh points in the channel (solid line). When fixing the number of mesh points in the channel as 20 (broken line), a real potential barrier was obtained. The difference in a maximum potential barrier between default and fixed meshes was $18 \mathrm{mV}$, which corresponded to the discontinuity in $V_{\text {th }}$. This is a typical example of the meshing noise.

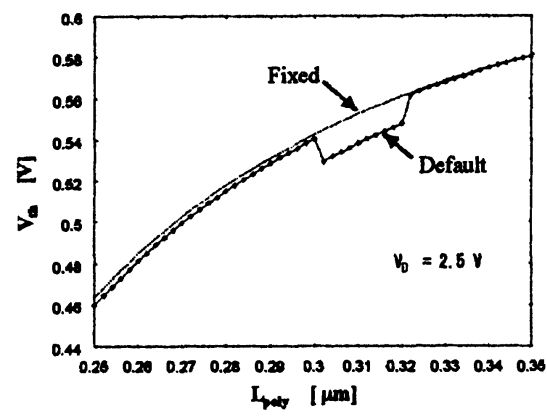

Fig. 7 shows the threshold voltage $V_{\text {t }}$ as a function of the channel length $L$ [9].

The mesh generation should be based on physics considering the relation between the mesh size and the physical quantities in order to reduce the meshing noise. Here, the mesh dependency of the inversion carrier density $\mathrm{N}_{\mathrm{inv}}[10]$ and the surface roughness mobility $\mu_{\text {surf }}[11]$ are discussed, which are very important for MOSFET simulation. 
The mesh dependency of $\mathrm{N}_{\mathrm{inv}}$ was investigated by Tanimoto and Shigyo [10]. Fig. 8 shows the $N_{i n v}-V_{G}$ characteristics for different grid size, where one-dimension simulation was used with constant grid size. Here, Delaunay and Voronoi meshes were used for the comparison. Device simulators have usually used Delaunay mesh, where the grid locates at the surface of the silicon substrate. On the other hand, the boundary of the control volume (CV) is locates at the surface for Voronoi mesh. As show in the Fig. 8, Delaunay mesh overestimated $\mathbf{N}_{\mathrm{inv}}$. Voronoi mesh underestimated it. These can be explained using Fig. 9. Delaunay mesh overestimated the number of carriers in the control volume at the surface, hence $\mathrm{N}_{\mathrm{inv}}$.

Fig. 10 shows minimum grid size to ensure the accuracy of $\mathrm{N}_{\mathrm{inv}}$ for Delaunay mesh. Less than $1 \mathrm{~nm}$ grid is required to ensure 10\% accuracy of $\mathrm{N}_{\mathrm{inv}}$ in the subthreshold region for the substrate concentration $N_{A}$ of $2 \times$ $10^{17} \mathrm{~cm}^{-3}$.

Fig. 11 shows the channel conductance as a function of grid size. Watt's model [12] considered the universality of $\mu_{\text {surf }}$ only by one girid at the surface. Thus, this model overestimated $\mu_{\text {surf }}$ with a decrease in the grid size, since the mobility at the next grid to the surface was considered to be bulk one. Shin et al. [13] proposed the method, which solved this problem. Their method provided a linkage between the local and effective mobility. Their method is mathematically correct, but it requires a fine mesh.

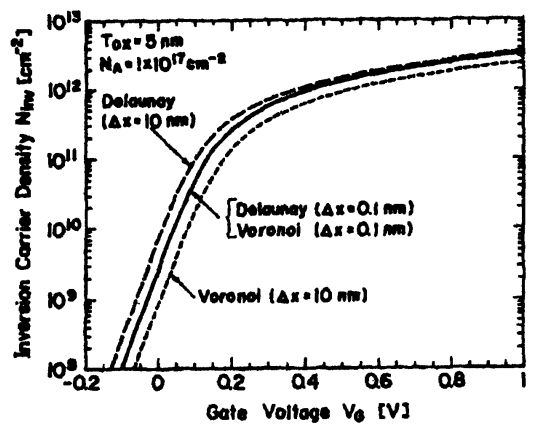

Fig. 8 Inversion carrier density $N_{\text {inv }}$ as a function of gate voltage $V_{O}[10]$.

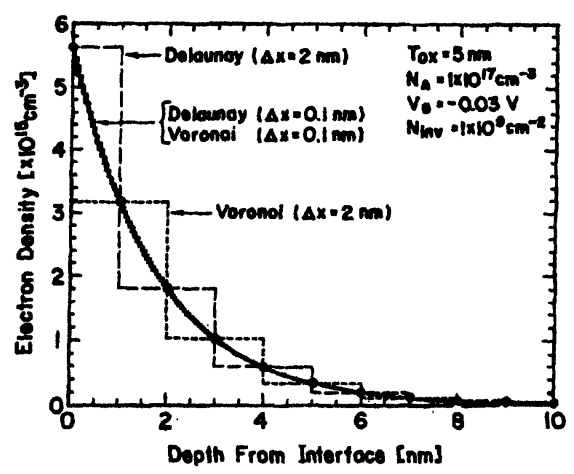

Fig. 9 Electron density as a function of depth [10]. Delaunay grid overestimated $N_{\text {inv }}$.

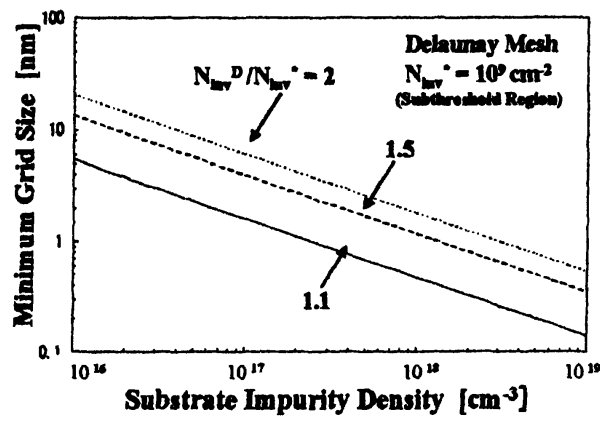

Fig. 10 Minimum grid size to ensure accuracy of $\mathrm{N}_{\text {inv }}$ for Delaunay mesh. 


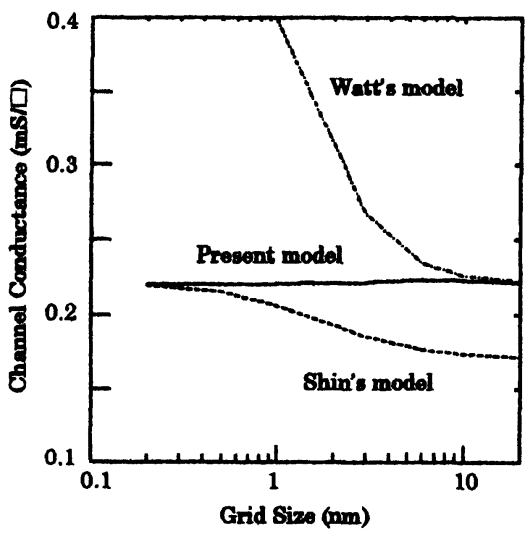

Fig. 11 Channel conductance as a function of grid size.

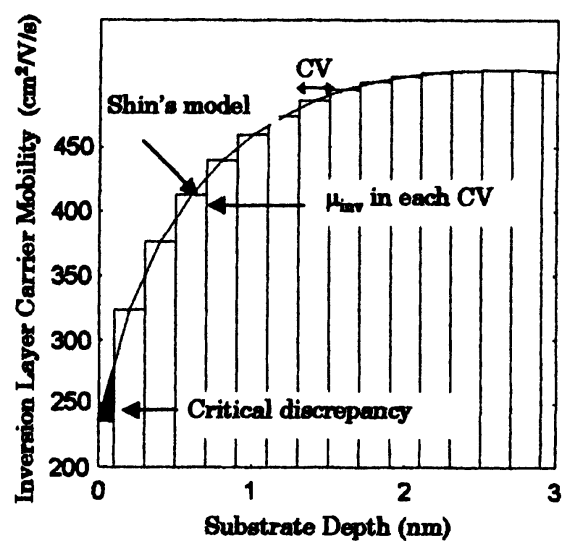

Fig. 12 Inversion carrier mobility as a function of depth [11].

Fig. 12 shows the cause of the grid size dependency. The major problem is the estimation of $\mu_{\text {aurf }}$ at the surface. Enda and Shigyo [11] modified Shin's model with physical consideration. The vertical electric field $E_{\mathrm{V}}$ in the surface control volume was estimated using the values of $E_{\mathrm{V}}$ at the surface and next grids. Using this formulation, the grid size dependence on $\mu_{\text {warf }}$ could be reduced, as shown in Fig. 11.

\section{Interconnect Variability}

The importance of the interconnect RC delay is becoming larger with the advance in VLSI. Fig. 13 shows the interconnect structure used for the analysis. Periodic lines [14] were used. In the analysis, the vertical scaling for the interconnect height $\mathrm{H}$ and thickness $T$ was not considered for the simplicity. A two-dimensional simulator [14] with $100 \times 70$ mesh points was used to calculate C.

Fig. 14 shows the relative variation of $\Delta \mathrm{C} / \mathrm{C}$ for $+10 \% \Delta W / W$. The space $S$ was equal to $\mathrm{W}-\Delta \mathrm{W}$. The parallel plate capacitance $\mathrm{C}_{\mathrm{P}}$ was calculated as $C_{P} /\left(K_{0 x} \varepsilon_{0} L\right) \equiv W / H+2$ $\mathrm{T} / \mathrm{S}$, where $\boldsymbol{K}_{\mathbf{0 x}}$ is dielectric constant of oxide, and $\varepsilon_{0}$ permittivity of free space. It is found that the simulated relative variation $\Delta C / C$ was smallest when $W / H \approx 2$, because of the fringing capacitance $C_{F}$, which is a parasitic component originated from the fringing

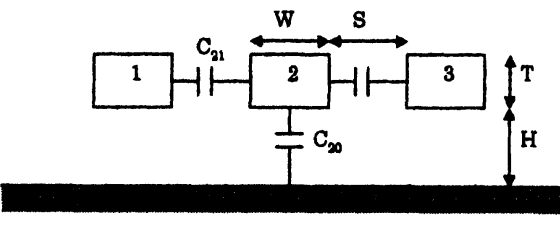

Fig. 13 Interconnect structure. $\mathrm{T} / \mathrm{H}=\mathbf{0 . 6}$.

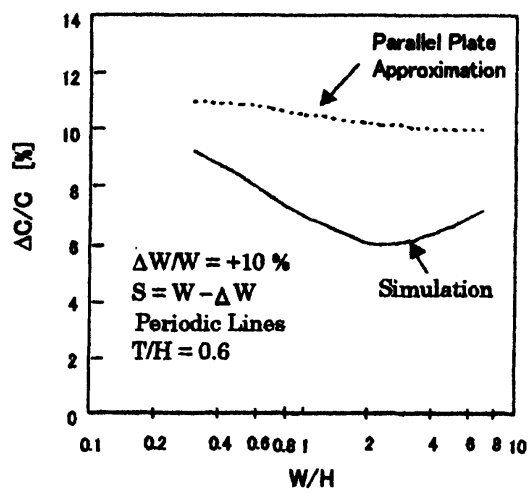

Fig. 14 Capacitance variation $\Delta C / C$ for $+10 \% C D$ variation, $\Delta W / W[15]$. 
electric field. $C_{F}$ reduces $\Delta C$.

Fig. 15 shows the relative variation of $|\Delta(\mathrm{RC}) /(\mathrm{RC})|$ for $+10 \% \Delta \mathrm{W} / \mathrm{W}$. Maximum variation occurred when $\mathrm{W} / \mathrm{H} \approx 2$, which was opposite to the minimum case for $\Delta \mathrm{C} / \mathrm{C}$. There is a tradeoff between $\Delta \mathrm{C} / \mathrm{C}$ and $\Delta(\mathrm{RC}) /(\mathrm{RC})$. The $\mathrm{RC}$ delay variation is decomposed as follows.

$$
\begin{aligned}
\frac{\Delta(\mathrm{RC})}{\mathrm{RC}} & =\frac{(\mathrm{R}+\Delta \mathrm{R})(\mathrm{C}+\Delta \mathrm{C})-\mathrm{RC}}{\mathrm{RC}} \\
& =\frac{\Delta \mathrm{R}}{\mathrm{R}}+\frac{\Delta \mathrm{C}}{\mathrm{C}}+\frac{\Delta \mathrm{R} \Delta \mathrm{C}}{\mathrm{RC}}
\end{aligned}
$$

In Fig. $15,|\Delta(\mathrm{RC}) /(\mathrm{RC})|$ was less than $1 \%$ for the parallel plate approximation. This is because the resistance variation $\Delta R / R$ was cancelled by $\Delta C / C$, i.e., there is a negative correlation between the two. In the actual case, $C_{F}$ reduced $\Delta C / C$, so that $\Delta R / R$ was not canceled by $\Delta C / C$. Fig. 16 shows each component of (1). The value of $\Delta R / R$ was constant so that the decrease in $\Delta C / C$ due to $C_{F}$ resulted in the increase in $|\Delta(\mathrm{RC}) /(\mathrm{RC})|$.

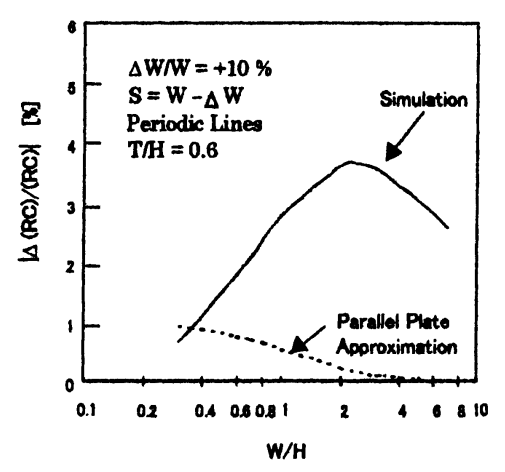

Fig. $15 R C$ delay variation $\Delta(R C) /(R C)$ for $+10 \% C D$ variation, $\Delta W / W$ [15].

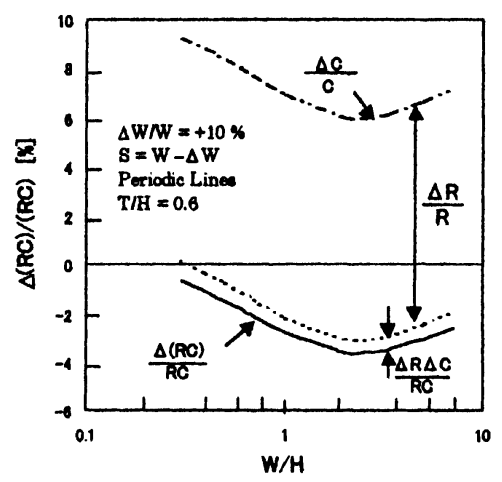

Fig. 16 Each component of (1) .

An interconnect design guideline to reduce $\mathrm{C}$ and/or $\mathrm{RC}$ delay variations is as follows. In the case of short interconnects, $\mathrm{R}_{\mathrm{tr}} \mathrm{C}$ is crucial for the circuit delay, so that the fringing ratio $F\left(\equiv \mathrm{C}_{\mathrm{F}} / \mathrm{C}_{\mathrm{P}}\right.$ where $\left.\mathrm{C}_{\mathrm{F}} \equiv \mathrm{C}-\mathrm{C}_{\mathrm{P}}\right)$ should be large to reduce the $\mathrm{C}$ variation. On the contrary, $F$ should be small for long interconnects to reduce the RC variation.

\section{Conclusion}

The robust designs of process, device and circuit are crucial for realize VLSI, since process fluctuations influence upon device and circuit performance. The statistical simulation using TCAD is one of methods to solve this problem. An example of the generation of MOSFET worst-case circuit models using TCAD was demonstrated. An analysis of the variability of the interconnect reveled that there is a tradeoff between $\Delta C / C$ and $\Delta(R C) /(R C)$ because of $C_{F}$. TCAD based statistical analysis become increasingly importance. The meshing noise should be solved with physical considerations. 


\section{Acknowledgements}

The author expresses sincere thanks to Dr. J. K. Kibarian and the members in PDF Solutions for fruitful discussions. I thank all of my colleagues in Toshiba Corporation who have contributed to this work.

\section{References}

[1] Director, S.W., Maly, W. (Ed.) (1994): Statistical approach to VLSI. NorthHolland.

[2] Jones, R.E., Mele, T.C. (1991): Use of screening and response surface measuremental designs for development of a $0.5-\mu \mathrm{m}$ CMOS self-aligned titanium silicide process. IEEE Trans. Semi. Manuf., 4: 281.

[3] Bakoglu, H.B., Meindl, J.D. (1985): Optimum interconnection circuits for VLSI. IEEE Trans. Electron Devices, ED-32: 903.

[4] Walker, D.M.H. et al. (1993): A TCAD framework for development and manufacturing. Technology CAD Systems (Ed. F. Fashing et al.): 83.

[5] Shigyo, N., et al. (2000): Technology CAD based statistical simulation of MOSFET's. Solid-St. Electronics, 44: 1001.

[6] Greenwood, J.A., Sandomire, M.M. (1950): Sample size required for estimating the standard deviation as a per cent of its true value. J. American Stat. Assoc., 45: 257.

[7] pdFab version 3.1 User's Reference Manual (1997): PDF Solutions.

[8] Shigyo, N. (2000): An analysis of process fluctuation induced propagation delay variation using analytical model: Solid-St. Electronics, 44: 2183.

[9] Shigyo, N., Tanimoto, H., Enda, T. (2000): Mesh related problems in device simulation: Treatments of meshing noise and leakage current. Solid-St. Electronics, 44: p. 11.

[10] Tanimoto, H., Shigyo, N. (1992): Discretization error in MOSFET device simulation. IEEE Trans. Computer Aided Design, 11: 921.

[11] Enda T., Shigyo, N. (1997): Grid size independent model of inversion layer carrier mobility. Proc. SISPAD'97: 319.

[12] Watt J.T., Plummer, J.D. (1987): Universal mobility-field curve for electrons and holes in MOS inversion layers. Symp. VLSI Tech. Dig. Papers: 81.

[13] Shin, H. et al. (1989): A new approach to verify and derive a transverse-fielddependent mobility model for electrons in MOS inversion layers. IEEE Trans. Electron Devices, 36: 1117.

[14] Shigyo, N., Fukuda, S., Kato, K. (1989): The influence of boundary locations on wiring capacitance simulation. IEEE Trans. Electron Devices, ED-36: 1171.

[15] Shigyo, N. (2000): Tradeoff between interconnect capacitance and RC delay variations induced by process fluctuations. IEEE Trans. Electron Devices, 47: 1740. 